\title{
Denoising Projection Data with a Robust Adaptive Bilateral Filter in Low-Count SPECT
}

\author{
Susumu Nakabayashi' ${ }^{1}$, Takashi Chikamatsu' ${ }^{2}$, Takao Okamoto ${ }^{3}$, Tatsuro Kaminaga ${ }^{4}$, \\ Norikazu Arai' ${ }^{2}$, Shinobu Kumagai'2, Kenshiro Shiraishi4, Takahide Okamoto ${ }^{1,2}$, \\ Takenori Kobayashi', Jun'ichi Kotoku' ${ }^{12^{*}}$
}

${ }^{1}$ Graduate School of Medical Care and Technology, Teikyo University, Tokyo, Japan

${ }^{2}$ Central Radiation Division, Teikyo University Hospital, Tokyo, Japan

${ }^{3}$ Diagnostic Imaging, PET Center, Musashimurayama Hospital, Tokyo, Japan

${ }^{4}$ Department of Radiology, Teikyo University School of Medicine, Tokyo, Japan

Email: ^kotoku@med.teikyo-u.ac.jp

How to cite this paper: Nakabayashi, S. Chikamatsu, T., Okamoto, T., Kaminaga, T., Arai, N., Kumagai, S., Shiraishi, K., Okamoto, T., Kobayashi, T. and Kotoku, J. (2018) Denoising Projection Data with a Robust Adaptive Bilateral Filter in Low-Count SPECT. International Journal of Medical Physics, Clinical Engineering and Radiation Oncology, 7, 363-375.

https://doi.org/10.4236/ijmpcero.2018.73030

Received: July 5, 2018

Accepted: August 17, 2018

Published: August 20, 2018

Copyright (c) 2018 by authors and Scientific Research Publishing Inc. This work is licensed under the Creative Commons Attribution-NonCommercial International License (CC BY-NC 4.0).

http://creativecommons.org/licenses/by-nc/4.0/ (c) (i) (8) Open Access

\begin{abstract}
Low-count SPECT images are well known to be smoothed strongly by a Butterworth filter for statistical noise reduction. Reconstructed images have a low signal-to-noise ratio (SNR) and spatial resolution because of the removal of high-frequency signal components. Using the developed robust adaptive bilateral filter (RABF), which was designed as a pre-stage filter of the Butterworth filter, this study was conducted to improve SNR without degrading the spatial resolution for low-count SPECT imaging. The filter can remove noise while preserving spatial resolution. To evaluate the proposed method, we extracted SNR and spatial resolution in a phantom study. We also conducted paired comparison for visual image quality evaluation in a clinical study. Results show that SNR was increased 1.4 times without degrading the spatial resolution. Visual image quality was improved significantly $(\mathrm{p}<0.01)$ for clinical low-count data. Moreover, the accumulation structure became sharper. A structure embedded in noise emerged. Our method, which denoises without degrading the spatial resolution for low-count SPECT images, is expected to increase the effectiveness of diagnosis for low-dose scanning and short acquisition time scanning.
\end{abstract}

\section{Keywords}

Bilateral Filter, Denoising, SPECT, Nuclear Medicine

\section{Introduction}

In recent years, dose reduction has attracted attention in many fields, e.g., 
pediatric examination in nuclear medicine. The image quality of nuclear medicine, however, largely depends on the acquisition count (dose and acquisition time) [1]. Dose reduction generally leads to deterioration in diagnostic quality because of the relative increase of noise components. In the Single photon emission computed tomography (SPECT) examination, image quality deterioration usually occurs as a result of limited acquisition time per projection. The increase of acquisition time is challenging due to the risk of motion artifacts.

Noise removal of raw SPECT projection data has been typically conducted by a low-pass filter to eliminate high-frequency noise which causes artifacts of SPECT reconstruction [2] [3] [4] [5] [6]. A Butterworth filter, which is usually used as a low-pass filter, however, cannot distinguish signal from noise. In low-count SPECT imaging, the Butterworth filter removes many high-frequency signal components along with noise and reduces the spatial resolution.

Retaining high-frequency signal components requires selective noise reduction for low-count SPECT imaging. This study specifically examined bilateral filters, which are extremely useful in Gaussian noise reduction with edge preservation [7]. Clinical image processing using this filter has been reported recently [8] [9] [10]. Nevertheless, the bilateral filter is known to perform poorly for very noisy images [11] [12].

Using a new filter algorithm based on the bilateral filter, we developed the robust adaptive bilateral filter (RABF), which has robust edge-preserving and smoothing effects for low-count data. We propose a new pre-processing method using the RABF as pre-stage filter of the Butterworth filter for low-count SPECT imaging. The low-count explained herein is a limited count such as that is used for pediatric examinations.

\section{Methods}

Butterworth filter, a high-frequency cut-off filter, is the standard choice of the pre-processing filter of SPECT image reconstructions. Frequency dependence of the Butterworth filter $B(f)$ is

$$
B(f)=\frac{1}{1+\left(\frac{f}{f_{\mathrm{c}}}\right)^{n}},
$$

where $n$ is the filter order and $f_{\mathrm{c}}$ is the cut-off frequency. $f_{\mathrm{c}}$ must be lowered to reduce large amounts of noise, but high-frequency signal components might thereby also be removed along with noise. A low-pass filter such as the Butterworth filter is generally effective to prevent artifacts, but it degrades image quality for low-count data.

We propose a new pre-processing method for low-count data using the robust adaptive bilateral filter (RABF) as a pre-stage filter of the Butterworth filter (Figure 1). Reducing noise in advance, the RABF prevents removal of signal components by the Butterworth filter. 


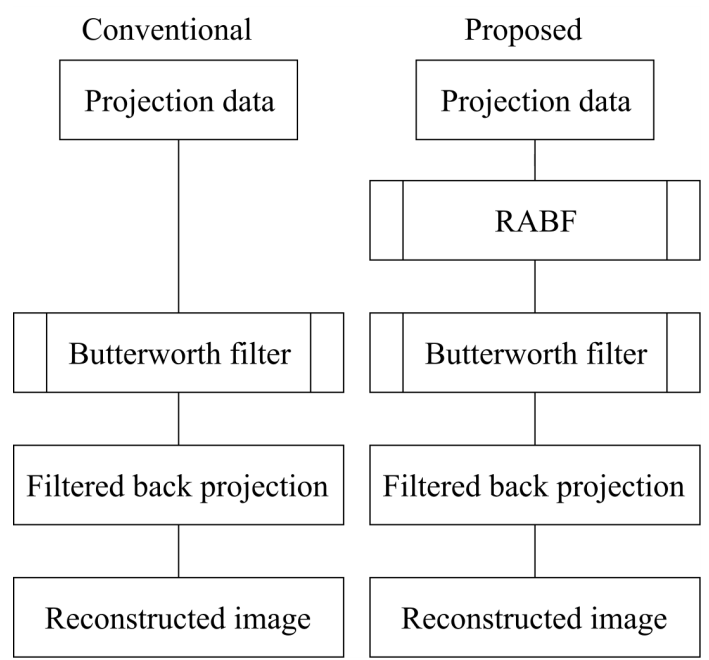

Figure 1. Flowcharts of the conventional and the proposed method.

\subsection{Bilateral Filter}

Bilateral filters are edge-preserving smoothing filters [7]. They have two Gaussian kernels which weigh the pixel distance and pixel value (intensity) difference between a center pixel and neighbouring ones. The bilateral filter is given as

$$
=\frac{\sum_{m=-w}^{w} \sum_{n=-w}^{w} f(i+m, j+n) \exp \left(-\frac{m^{2}+n^{2}}{2 \sigma_{s}^{2}}\right) \exp \left\{-\frac{[f(i+m, j+n)-f(i, j)]^{2}}{2 \sigma_{I}^{2}}\right\}}{\sum_{m=-w}^{w} \sum_{n=-w}^{w} \exp \left(-\frac{m^{2}+n^{2}}{2 \sigma_{s}^{2}}\right) \exp \left\{-\frac{[f(i+m, j+n)-f(i, j)]^{2}}{2 \sigma_{I}^{2}}\right\}}
$$

where $g(i, j)$ represents the filtered pixel value at pixel $(i, j)$. In addition, $f(i, j)$ denotes the original pixel value. $\sigma_{s}$ signifies the standard deviation of spatial Gaussian kernel for smoothing. $\sigma_{I}$ stands for the standard deviation of intensity Gaussian kernel for edge-preservation.

The bilateral filter distinguishes between edges and noise based on their difference in intensity. This filter smoothes intensity variations smaller than $\sigma_{I}$ as noise and preserves intensity differences greater than $\sigma_{I}$ as edges. For noisy images as SPECT projection data, the intensity of the Gaussian kernel does not function well because the intensity difference itself includes large amounts of noise. Outliers with large intensity difference are preserved. Edges buried in noise are smoothed where the difference is slight.

\subsection{Robust Adaptive Bilateral Filter (RABF)}

This newly developed filter achieves robust noise reduction and edge-preservation, even in noisy images. Retaining a general algorithm of the bilateral filter in Equation (2), the RABF replaces $f(i, j)$ with the mean value $\mu(i+\hat{k}, j+\hat{l})$ as a measure against outliers. 
This new parameter is calculated over the $3 \times 3$ kernel centered on pixel $(i+\hat{k}, j+\hat{l})$, which was selected from the pixel and its eight surrounding pixels to obtain the stable intensity difference without adverse effects by outliers. Therein, $\hat{k}$ and $\hat{l}$ are given as

$$
\hat{k}, \hat{l}=\arg \min _{k, l \in A} \sum_{s, t \in A}[f(i+k+s, j+l+t)-f(i, j)]^{2},
$$

where $A$ is $\{-1,0,1\}$. The flow of determining the calculated area is shown in (Figure 2). The sum of the squares of the differences from the processing pixels in the $3 \times 3$ kernel is calculated from each of the nine areas. Pixel $(i+\hat{k}, j+\hat{l})$ is the center of the kernel in which the sum of squares of the difference is minimized among them. The kernel with the minimum computed value is regarded as not including outliers. Stable parameters are obtainable using the values in it.

This filter can also adopt the standard deviation value $\sigma_{I}(i+\hat{k}, j+\hat{l})$ from the same kernel as intensity weight $\sigma_{I}$. The selected kernel avoids outliers and edges. An example of new parameter determination is presented in Figure 3.

\begin{tabular}{|c|c|c|c|c|}
\multicolumn{6}{c|}{ Pixel values of $5 \times 5$} \\
\hline 4 & 5 & 7 & 8 & 9 \\
\hline 6 & 7 & 8 & 10 & 1 \\
\hline 10 & 9 & \begin{tabular}{c}
10 \\
\hline$(i, j)$
\end{tabular} & 9 & 11 \\
\hline 12 & 15 & 8 & 14 & 13 \\
\hline 25 & 14 & 13 & 10 & 12 \\
\hline
\end{tabular}

sums of squares of the difference between $(i, j)$ of 9 areas $(3 \times 3)$

\begin{tabular}{|l|l|l|l|l|}
\hline & & & & \\
\hline & 100 & 53 & 101 & \\
\hline & 63 & $\begin{array}{c}60 \\
(i, j)\end{array}$ & 116 & \\
\hline & 284 & 72 & $\begin{array}{c}44 \\
\text { min }\end{array}$ & \\
\hline & & \multicolumn{2}{|c|}{$\frac{\hat{k}=+1, \hat{l}=+1}{\perp}$} \\
\hline
\end{tabular}

Figure 2. Search area for the minimum sum of squares of the difference between the processing pixel for ascertaining stable parameters (Equation (3)). The left kernel shows pixel values of $5 \times 5$. Values of the right kernel are the calculated results of nine sums of squares of the difference. For example, the left and right green frames and blue frames show calculation areas and results. The value of the red frame is the minimum value. Its position pixel $(i+\hat{k}, j+\hat{l})$ is the center $3 \times 3$ for obtaining the new parameters.

mean and standard deviation of the area $(3 \times 3)$ centered on pixel $(i+\hat{k}, j+\hat{l})$

Pixel values

\begin{tabular}{|c|c|c|c|c|c|}
\hline 4 & 5 & 7 & 8 & 9 & \multirow{2}{*}{$\hat{k}=+1, \hat{l}=+1$} \\
\hline 6 & 7 & 8 & 10 & 1 & \\
\hline 10 & 9 & 10 & 9 & 11 & $\mu(i+\hat{k}, j+\hat{l})=11.1$ \\
\hline 12 & 15 & 8 & 14 & 13 & $\sigma_{I}(i+\hat{k}, j+\hat{l})=2.03$ \\
\hline 25 & 14 & 13 & 10 & 12 & \\
\hline
\end{tabular}

Figure 3. The area obtained in Figure 2 is shown within the red frame. $\mu(i+\hat{k}, j+\hat{l})$ and $\sigma_{I}(i+\hat{k}, j+\hat{l})$ respectively represent the mean and standard deviation of the red frame values. In this kernel, we set pixel values 1 and 25 as outliers and set the upper left area as an edge. 
The resulting $\mathrm{RABF}$ is given as

$$
\begin{aligned}
& g(i, j) \\
& =\frac{\sum_{m=-2}^{2} \sum_{n=-2}^{2} f(i+m, j+n) \exp \left(-\frac{m^{2}+n^{2}}{2 \sigma_{s}^{2}}\right) \exp \left\{-\frac{[f(i+m, j+n)-\mu(i+\hat{k}, j+\hat{l})]^{2}}{2 \sigma_{I}^{2}(i+\hat{k}, j+\hat{l})}\right\}}{\sum_{m=-2}^{2} \sum_{n=-2}^{2} \exp \left(-\frac{m^{2}+n^{2}}{2 \sigma_{s}^{2}}\right) \exp \left\{-\frac{[f(i+m, j+n)-\mu(i+\hat{k}, j+\hat{l})]^{2}}{2 \sigma_{I}^{2}(i+\hat{k}, j+\hat{l})}\right\}}
\end{aligned}
$$

where the kernel size in this study is $5 \times 5$ and $\sigma_{s}=1$. New parameters are updated for each processing pixel.

The Gaussian intensity kernel of RABF operates sufficiently well using $\mu(i+\hat{k}, j+\hat{l})$ instead of $f(i, j)$ if the processing pixels have an outlier (Figure 4). Contrary to the weighting of the bilateral filter, that of the RABF is independent of outliers. Therefore, the RABF works robustly even for images with outliers.

\subsection{Phantom Study}

We assessed the RABF to confirm that it works on SPECT projection data as expected. We also compared the results to those obtained using the bilateral filter. Then, using a digital phantom to evaluate image quality, we extracted the spatial resolution and SNR as an objective evaluation.

\subsubsection{Phantom Data and Data Processing}

To evaluate image quality objectively, we extracted the spatial resolution and SNR with a digital phantom. We used a digital brain phantom produced by software (Prominence Processor Ver.3.1; Prominence Conference, Japan) [13]. The total counts were 3.4 million and other collection conditions are presented in Table 1. We also prepared reference data with high-count (total counts were about 100 million) for image evaluation. The simulated phantom data are portrayed in Figure 5. We respectively designate pre-processing methods with

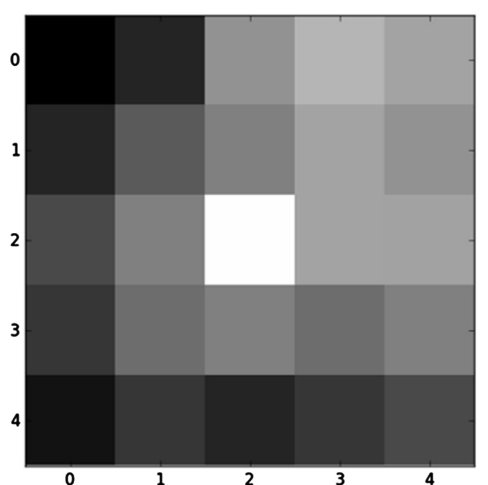

(a)

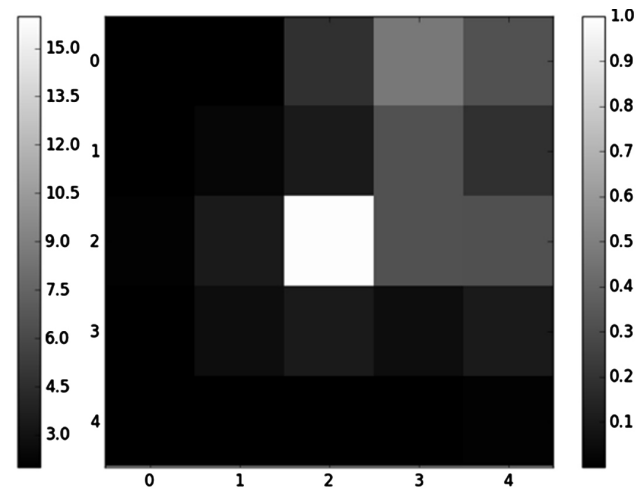

(b)

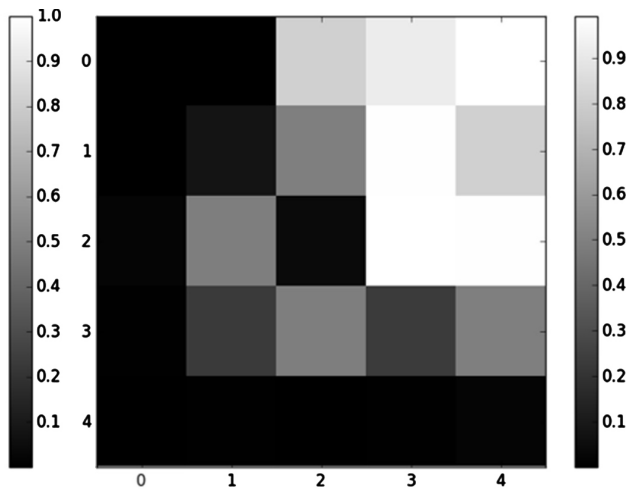

(c)

Figure 4. Example of intensity weighting when processing pixel is the outlier: (a) pixel values of the kernel $(5 \times 5)$ for which the center is an outlier, (b) the intensity weight map by the bilateral filter for (a), and (c) the intensity weight map by RABF for (a). 
Table 1. Collection conditions of the simulated brain digital phantom.

\begin{tabular}{cc}
\hline Collection condition & Parameter \\
Matrix size & $128 \times 128(2 \mathrm{~mm} /$ pixel $)$ \\
Collection angles & $4^{\circ}(90 \mathrm{step})$ \\
Radius of gyration & $13 \mathrm{~cm}$ \\
System resolution FWHM at $13 \mathrm{~cm}$ & $8 \mathrm{~mm}$ \\
Counts & $30 \mathrm{k}$ counts/projection \\
Ratio of concentration & $4: 1$ (Gray matter:White matter $)$ \\
\hline
\end{tabular}

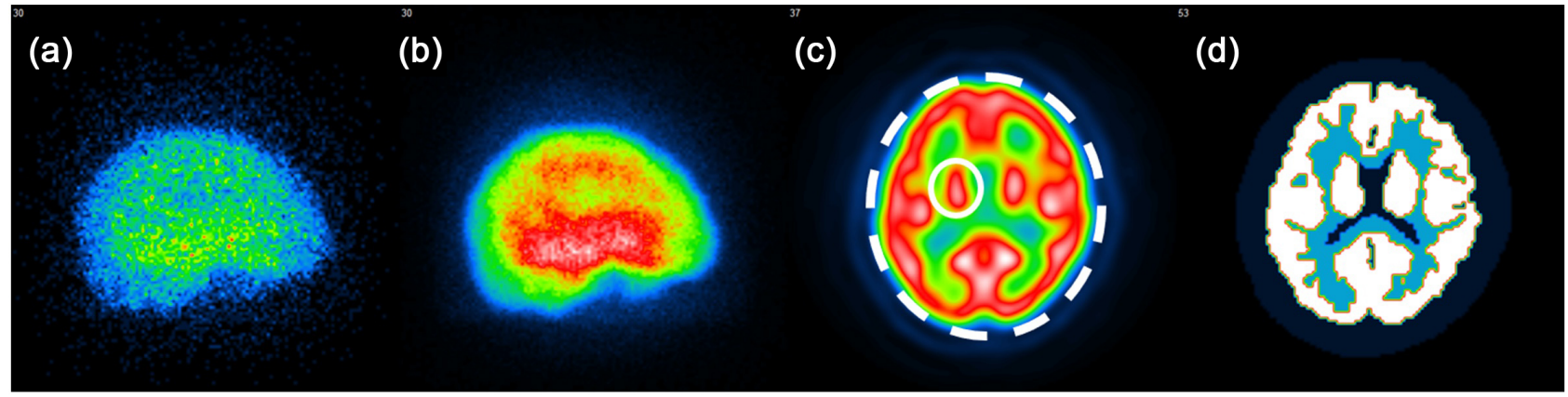

Figure 5. Simulated brain digital phantoms: (a) low-count projection data, (b) high-count projection data, and (c) a reconstructed image of basal ganglia level from (b). A white ellipse indicates the striatum where we calculated the FWHM. The standard deviation of noise was obtained from the region outside of the dotted ellipse. These phantoms were simulated based on (d). The color scale is Rainbow White.

both RABF and Butterworth filtering and with single Butterworth filtering as the RABF and conventional methods (Figure 1).

We used filtered back projection which is routinely used for SPECT reconstruction. The value of $f_{\mathrm{c}}$ was found based on the normalized mean square error (NMSE) between reference images and reconstructed images [2] [3]. NMSE is defined as [13]

$$
\mathrm{NMSE}=\frac{\sum_{i=1}^{N} \sum_{j=1}^{128} \sum_{k=1}^{128}[T(i, j, k)-R(i, j, k)]^{2}}{\sum_{i=1}^{N} \sum_{j=1}^{128} \sum_{k=1}^{128} R(i, j, k)^{2}},
$$

where $N$ represents the number of images, and where $R(i, j, k)$ and $T(i, j, k)$ respectively denote the normalized pixel value at $(i, j, k)$ in reference and reconstructed images. Optimal $f_{c}$ was found as the frequency at which NMSE was minimized.

\subsubsection{Objective Evaluation}

We evaluated the full width at half maximum (FWHM) from the fitting curve around the striatum (the ellipse in Figure $5(\mathrm{c})$ ) as the spatial resolution. SNR was obtained as

$$
\mathrm{SNR}=20 \log \frac{\mathrm{SD}_{\text {image }}}{\mathrm{SD}_{\text {noise }}}(\mathrm{dB}),
$$

where $\mathrm{SD}_{\text {image }}$ represents the standard deviation of all pixel values. Also, 
$\mathrm{SD}_{\text {noise }}$ represents the standard deviation of the peripheral region of the brain on the image (outside of the dotted ellipse in Figure 5(c)).

\subsection{Clinical Study}

To evaluate the usefulness of the RABF method for clinical data, two patient data with different acquisition count were compared. We examined significant differences in the visual inspection using the paired comparison method to verify the visual effects of RABF method. All data were anonymized before analysis.

\subsubsection{Patient Data and Data Processing}

The SPECT projection data were ${ }^{9{ }^{9} \mathrm{~m}} \mathrm{Tc}$ ethyl cysteinate dimer $\left({ }^{99 \mathrm{~m}} \mathrm{Tc}-\mathrm{ECD}\right)$ SPECT scan data of cerebral blood flow pattern measurements from two patients at Teikyo University Hospital. We used data only after anonymization. We found a low dose patient having an approximate weight of adults. To compare with this data, we prepared normal dose data with similar weight and collection conditions. Their disease types were intermittent epilepsy and suspected dementia with Lewy bodies, respectively, and there were no abnormal findings. The two patients, an adolescent (men, 17 years old, $60 \mathrm{~kg}$ ) and an adult (female, 76 years old, $65 \mathrm{~kg}$ ), had been respectively administered 350 and $840 \mathrm{MBq}$ doses. The total counts were respectively 2.3 million and 6.5 million. We respectively designated the data of the adolescent and adult as low and normal dose data. Other conditions are presented in Table 2.

The optimum $f_{\mathrm{c}}$ was not known for clinical images. Therefore, we adopted three $f_{\mathrm{c}}$ values for each datum as following: $(0.45,0.50$, and $0.55 \mathrm{cycles} / \mathrm{cm})$ and $(0.55,0.60$, and $0.65 \mathrm{cycles} / \mathrm{cm})$ for low-dose and normal data, respectively. Table 3 shows the $f_{\mathrm{c}}$ values used for low-dose and normal-dose projections, where $\mathrm{L}$, $\mathrm{N}, \mathrm{R}$, and $\mathrm{C}$ denote low-dose, normal dose, RABF and conventional method, respectively. In addition, $\mathrm{R}$ and $\mathrm{C}$ respectively represent the RABF and conventional method. Subscripts signify the used $f_{\mathrm{c}}$. Here, the filter order of the Butterworth filter was 8. Six images obtained for each datum are presented in Figure 6.

Table 2. Collection conditions for clinical images.

\begin{tabular}{cc}
\hline Collection condition & Parameter \\
\hline$\gamma$ camera & e.cam signature (Toshiba Corp.) \\
Collimator & LEHR (FWHM: $7.4 \mathrm{~mm})$ \\
Energy window & $140 \mathrm{keV} \pm 10 \%$ \\
Collection angles, mode & $4^{\circ}(90 \mathrm{step})$, Continuous mode \\
Acquisition time & $16 \mathrm{~min}$ \\
Turning radius & About $13.0-15.0 \mathrm{~cm}$ \\
Matrix size & $128 \times 128(2.1 \mathrm{~mm} / \mathrm{pixel})$ \\
Attenuation correction & Chang algorithm, $\mu=0.09 \mathrm{~cm}^{-1}$ \\
Color scale, window width & Rainbow White, $100 \%-0 \%$ \\
\hline
\end{tabular}


Table 3. Evaluation objects for each condition.

\begin{tabular}{ccccccc}
\hline & \multicolumn{2}{c}{ Low dose images $(350 \mathrm{MBq})$} & \multicolumn{2}{c}{ Normal dose images $(840 \mathrm{MBq})$} \\
\hline $\boldsymbol{f}_{\mathrm{c}}(\mathrm{cycles} / \mathrm{cm})$ & 0.45 & 0.50 & 0.55 & 0.55 & 0.60 & 0.65 \\
RABF + Butterworth & $\mathrm{L}\left(R_{0.45}\right)$ & $\mathrm{L}\left(R_{0.50}\right)$ & $\mathrm{L}\left(R_{0.55}\right)$ & $\mathrm{N}\left(R_{0.55}\right)$ & $\mathrm{N}\left(R_{0.60}\right)$ & $\mathrm{N}\left(R_{0.65}\right)$ \\
Butterworth & $\mathrm{L}\left(C_{0.45}\right)$ & $\mathrm{L}\left(C_{0.50}\right)$ & $\mathrm{L}\left(C_{0.55}\right)$ & $\mathrm{N}\left(C_{0.55}\right)$ & $\mathrm{N}\left(C_{0.60}\right)$ & $\mathrm{N}\left(C_{0.65}\right)$ \\
\hline
\end{tabular}

(a)
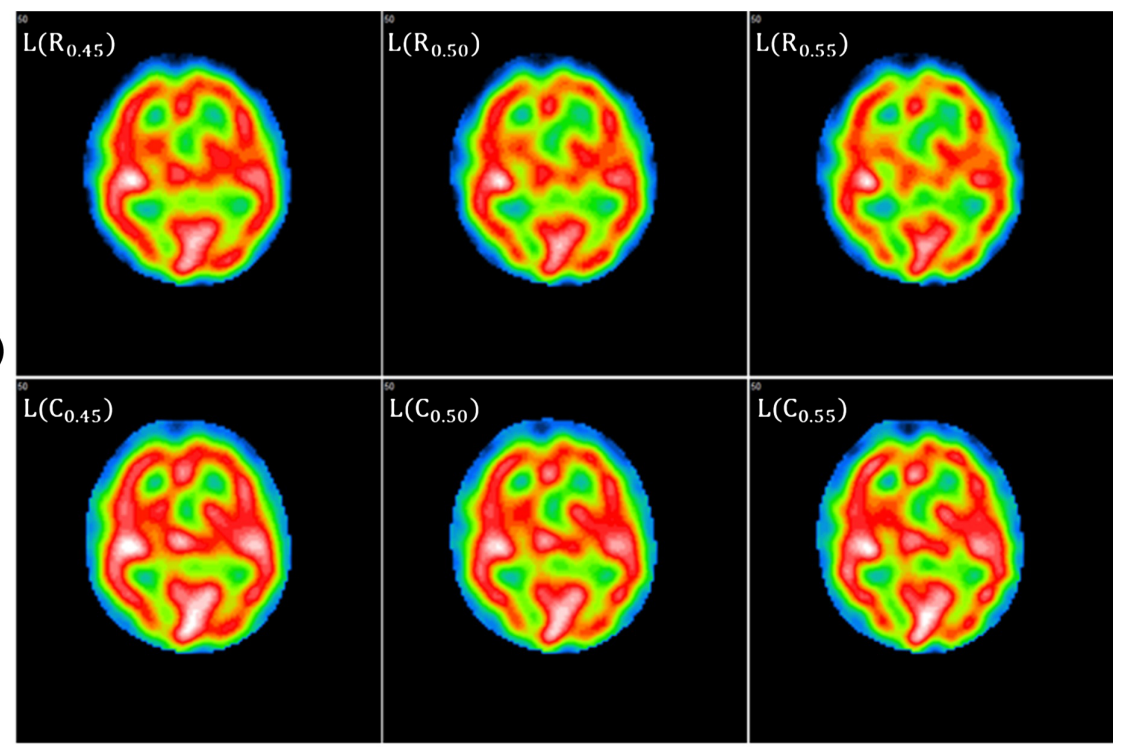

(b)
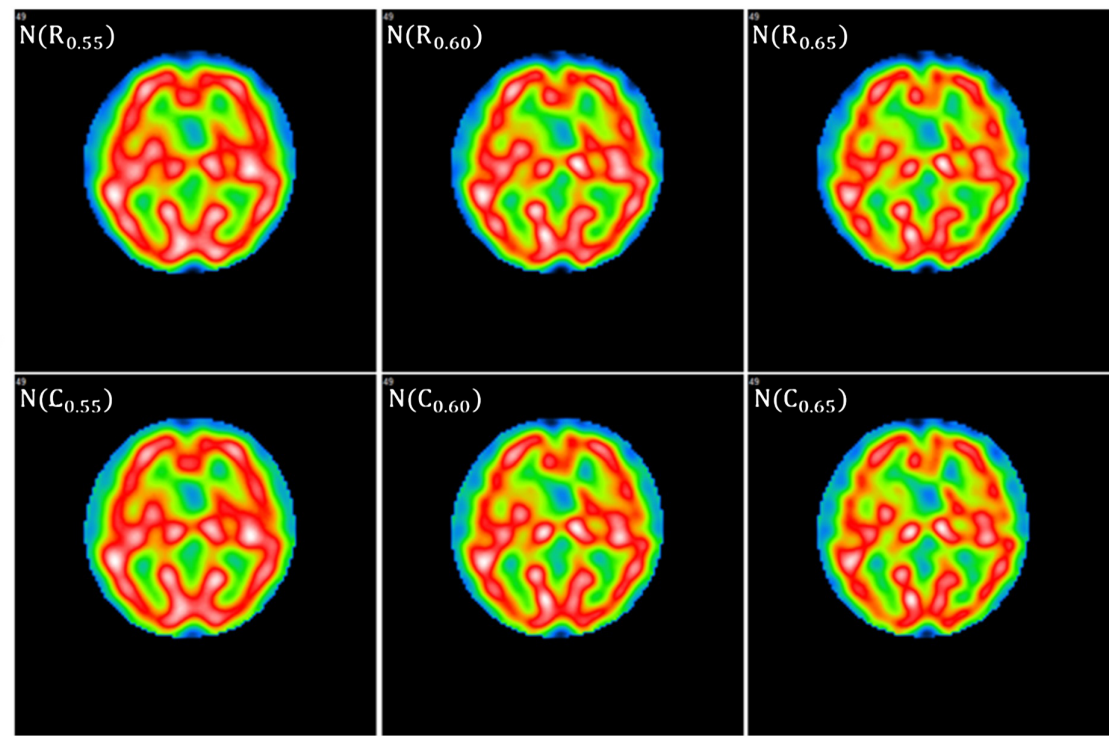

Figure 6. Objects of subjective evaluation: (a) low-dose images and (b) normal dose images. Upper and lower rows respectively present images reconstructed using RABF and the conventional method. $\mathrm{L}, \mathrm{N}, \mathrm{R}$, and $\mathrm{C}$ are abbreviations for low, normal, RABF, and conventional. The numbers $0.55-0.65$ are the cut-off values.

\subsubsection{Subjective Evaluation}

We compared the visual image quality of reconstructed images with those obtained with the conventional and the RABF method using Scheffe's method of paired comparison (modified method by Ura) [14] [15]. In Ura's method, all 
subjects compare all image combinations considering the order effect. When the number of objects is large, the evaluation reliability decreases because the burden on subjects becomes heavy. In this respect, this method is expected to present reliable results because only 30 objects are to be evaluated for six images.

The psychological scale of image quality comparison has five grades ( -2 to 2 , 0 means equality). Here, the image quality was defined as the visibility of the accumulated structure, which depends on the noise and spatial resolution. The subjects were 2 doctors and 18 radiological technologists. This evaluation was conducted with one display without looking back. We calculated the average preference score of every object using analysis of variance.

We also ascertained whether significant differences were found among the objects using yardstick $Y$, which is given as

$$
Y_{\varnothing}=q_{\varnothing}\left(t, f_{e}\right) \sqrt{\frac{V_{e}}{t n}},
$$

where $\varnothing$ represents a significance level of 0.05 or $0.01, t$ signifies the number of objects, $n$ denotes the number of subjects, and $f_{\mathrm{e}}$ and $V_{\mathrm{e}}$ respectively stand for the degrees of freedom and a variance of errors. Now, $q_{\varnothing}\left(t, f_{e}\right)$ is obtained from the Studentized range distribution. Significant difference between the two evaluation objects was inferred if the absolute value of difference among the average preference scores was found to be higher than $Y$.

\section{Results}

\subsection{Phantom Study}

Filtered projection data obtained using RABF and the bilateral filter are shown in Figure 7. The accumulation structure buried in noise emerged by RABF, but little change was found in the image filtered using the bilateral filter.

Resulting images with optimum $f_{\mathrm{c}}$ are depicted in Figure 8. The used $f_{\mathrm{c}}$ of the conventional and RABF method were, respectively, 0.55 and $0.60 \mathrm{cycles} / \mathrm{cm}$.

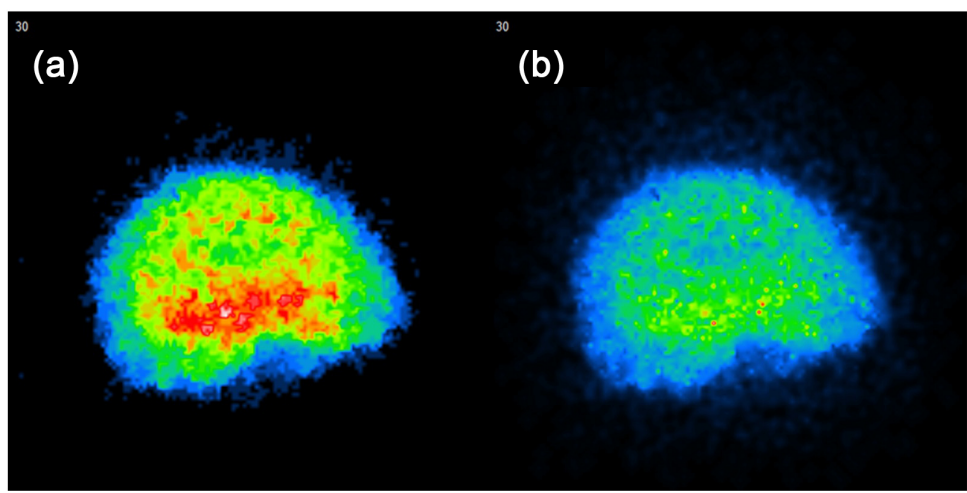

Figure 7. Influence of RABF and the bilateral filter for low-count SPECT projection data in Figure 5(a). Panels (a) and (b) respectively portray the filtered low-count data obtained using the RABF and the bilateral filter. The kernel size and $\sigma_{s}$ of these filters were, respectively, $5 \times 5$ and $\sigma_{s}=1$ pixel. Parameter $\sigma_{I}$ of bilateral filter was the standard deviation in all pixels. 
Here, the filter order was 8. Although the RABF method used higher $f_{c}$, the noise became less than that obtained using the conventional method.

Figure 9 shows the profile curve of the dashed line in Figure 8. The difference between the peak and valley of accumulation was increased slightly using the RABF method. The FWHM of the striatum (ellipse in Figure 5(c)) was improved slightly when using the RABF method: $29.0 \mathrm{~mm}$ vs. $28.7 \mathrm{~mm}$. The SNR was significantly higher when using the RABF method: $20.59 \mathrm{~dB}$ vs. $23.59 \mathrm{~dB}$. Comparison to results obtained using the conventional method demonstrates that the RABF method reduced noise considerably without degrading the spatial resolution.

\subsection{Clinical Study}

Visual evaluation indicated improvement by the RABF method for each $f_{\mathrm{c}}$ in low-dose images (Figure 10(a)). As presented in Figure 6(a), the accumulation structure became sharper when using RABF method. The best average preference scores were 0.283 obtained with the RABF method $L\left(R_{0.50}\right)$ and 0.042

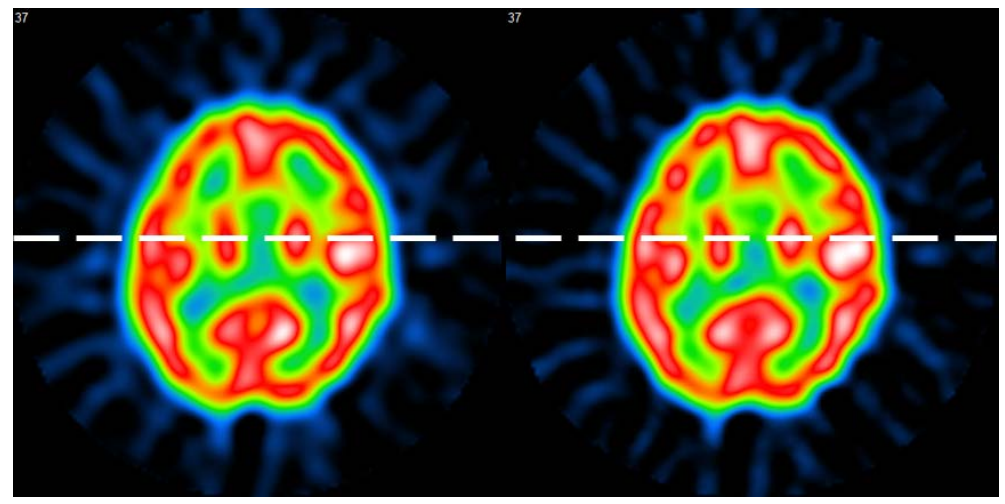

Figure 8. Reconstructed images from Figure 5(a) using the conventional (left) or the RABF method (right) with optimum $f_{c}(0.55$ and 0.60 cycles $/ \mathrm{cm}$, respectively).

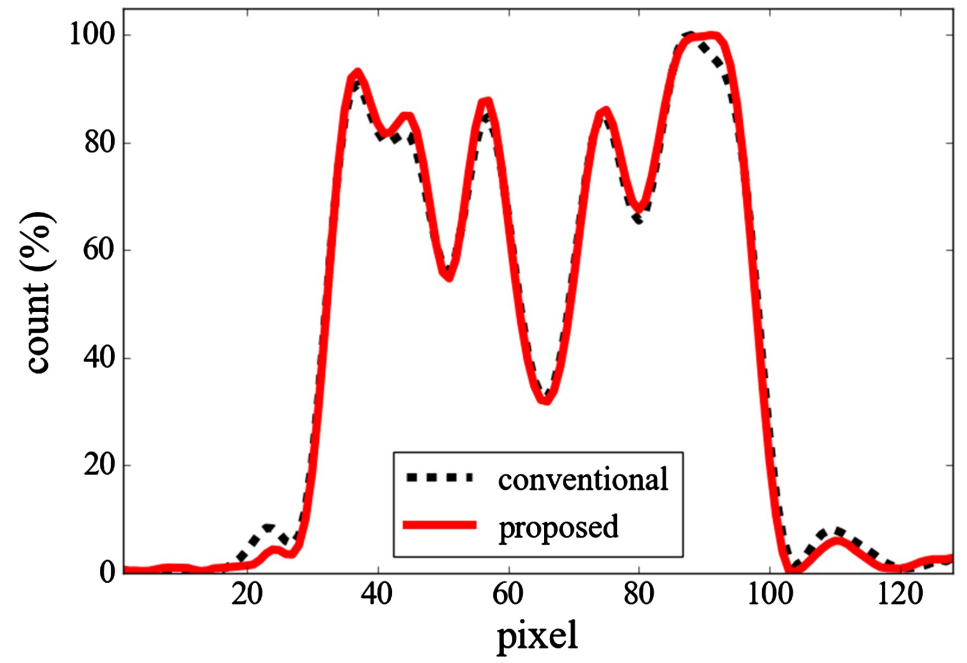

Figure 9. Profile curve of the dashed line in Figure 8. Each count (pixel value) was normalized by the maximum value. 
using the conventional method $\mathrm{L}\left(\mathrm{C}_{0.55}\right)$.

Reconstructed low-dose images $\mathrm{L}\left(\mathrm{C}_{0.55}\right)$ and $\mathrm{L}\left(\mathrm{R}_{0.50}\right)$ in Figure 11 show two profile curves along the line through the striatum. Comparison of the two profile curves reveals that the RABF method clarified the peaks of accumulation areas. Moreover, a buried peak of the striatum emerged. The RABF method significantly improved image quality $\left(p<0.01: Y_{0.05}=0.170, Y_{0.01}=0.200\right)$.

However, not as much difference as we had expected was found in normal dose images (Figure 6(b)). No significant difference was found in the results presented in Figure 10(b) $\left(p>0.05: Y_{0.05}=0.187, Y_{0.01}=0.219\right)$.

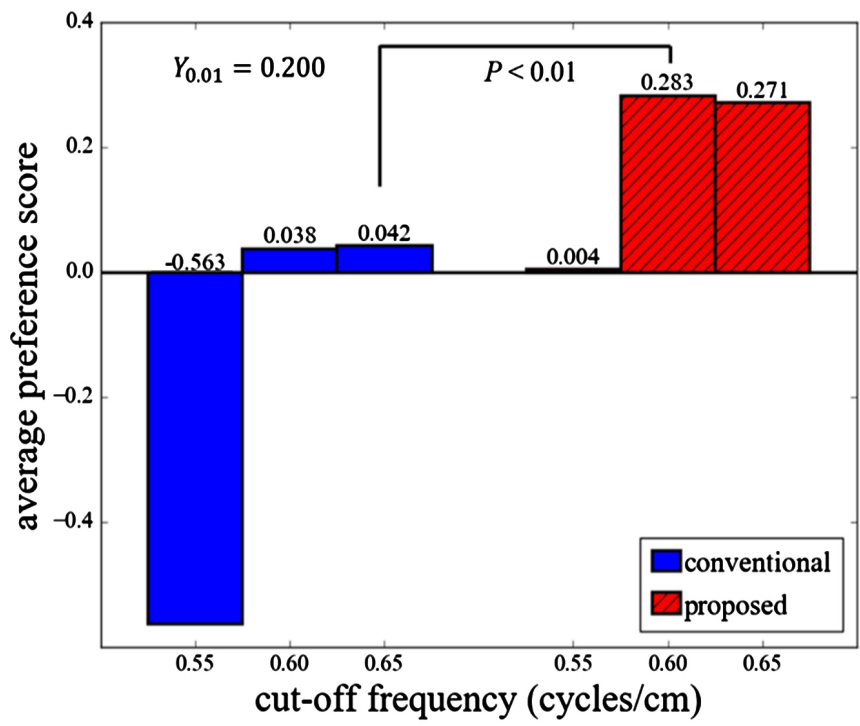

(a)

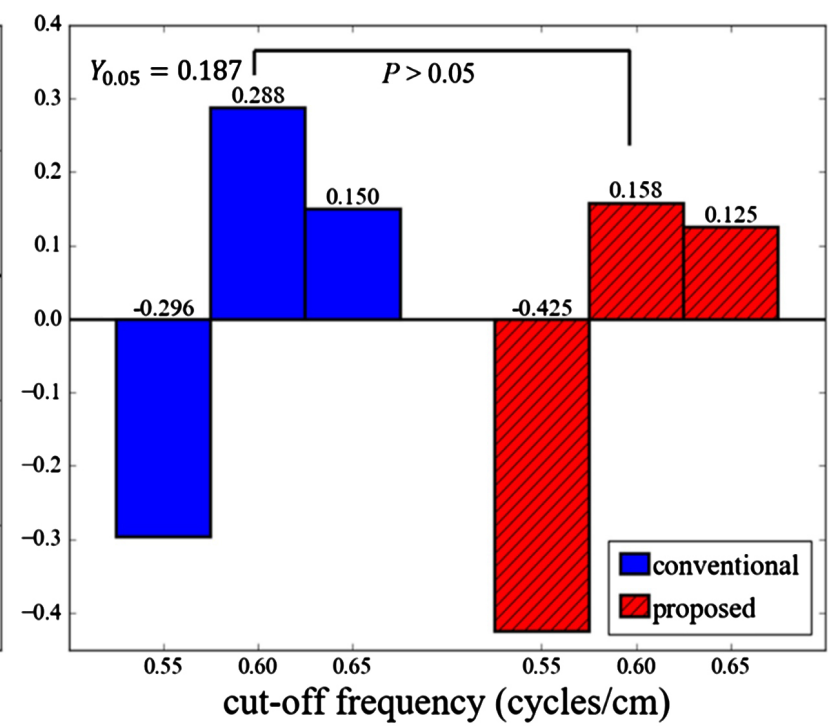

(b)

Figure 10. Average preference score: (a) low-dose image test and (b) normal dose image test. Presence or absence of significant difference of the maximum of average preference scores is shown for the conventional and RABF methods.

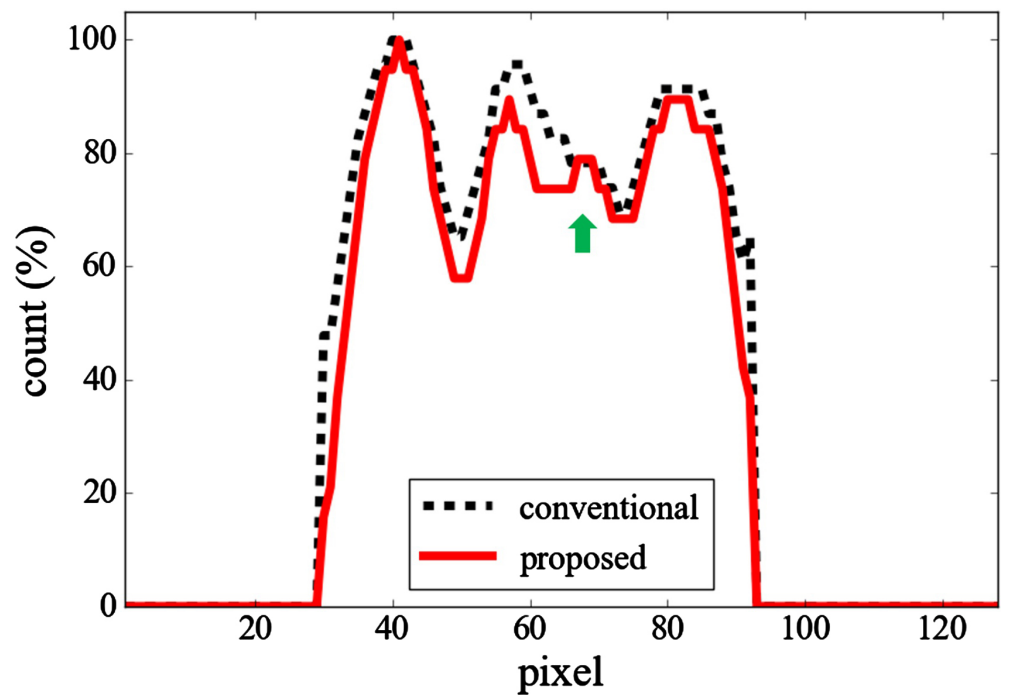

Figure 11. Profile curves of the line through the striatum at $\mathrm{L}\left(\mathrm{C}_{0.55}\right)$ and $\mathrm{L}\left(\mathrm{R}_{0.50}\right)$ in Figure 6. The two peaks of the striatum were confirmed using the RABF method. A buried peak emerged at 67 pixels. 


\section{Discussions}

Use of the Butterworth filter alone degrades image quality considerably for low-count SPECT data because it removes high-frequency signal components along with noise. Using RABF, we strove to remove noise without degrading the spatial resolution for low-count SPECT imaging.

The RABF worked robustly even with noisy images from SPECT projection data. This result cannot be obtained with the bilateral filter (Figure 7).

Our phantom study revealed that the RABF improved SNR without degrading the spatial resolution. The SNR was increased considerably (1.4 times) by the RABF method despite using high $f_{c}$. This result indicates that the RABF method has the same effect of doubling the count in nuclear medicine. It is expected to be very effective for low-count clinical practice.

Significant $(p<0.01)$ difference in visual image quality was confirmed from this clinical study. Visual observation shows that the accumulation structure was clarified (Figure 6(a)). The RABF was able to remove the noise so that the buried peak of striatum emerged in Figure 11. Normal dose images, however, showed little change in visual image quality. As confirmed from the phantom study, image quality improvement occurred because of strong noise reduction of the RABF. For that reason, the RABF method was not necessarily useful for high-count data because strong smoothing was not required.

\section{Conclusion}

We propose a new pre-processing method using RABF as a pre-stage filter for denoising without degrading the spatial resolution of low-count SPECT data, such as those from pediatric examinations. This filter, which had robust edge-preserving and smoothing effects, produced the benefits of improving SNR while preserving the spatial resolution. Results of a clinical study demonstrated that the RABF method clarified the accumulation structure that had been obscured within a considerable amount of noise. This method is expected to be adequate for low-dose scanning and short acquisition time scanning.

\section{Acknowledgements}

This work was partly supported by a Japan Society for the Promotion of Science (JSPS) KAKENHI Grant (No. 18K07646).

\section{Conflicts of Interest}

The authors declare no conflicts of interest regarding the publication of this paper.

\section{References}

[1] Groch, M.W. and Erwin, W.D. (2000) SPECT in the Year 2000: Basic Principles. Journal of Nuclear Medicine Technology, 28, 233-244.

[2] Minoshima, S., Maruno, H., Yui, N., Togawa, T., Kinoshita, F., Kubota, M., et al. 
(1993) Optimization of Butterworth Filter for Brain SPECT Imaging. Annals of Nuclear Medicine, 7, 71-77. https://doi.org/10.1007/BF03164571

[3] David, R.G., Gilland, A.B., Yaghoobi, N., Firouzabady, H. and Rustgou, F. (1988) Determination of the Optimum Filter Function for SPECT Imaging. Journal of Nuclear Medicine, 29, 643-650.

[4] Lyra, M. and Ploussi, A. (2011) Filtering in SPECT Image Reconstruction. International Journal of Biomedical Imaging, 2011, Article ID: 693795. https://doi.org/10.1155/2011/693795

[5] Matsutomo, N., Tanaka, T., Nagaki, A. and Sasaki, M. (2014) Validation of Noise Reduction in Iterative Reconstruction: A Simulation Phantom Study. Nihon Hoshasen Gijutsu Gakkai Zasshi, 70, 773-783. https://doi.org/10.6009/jjrt.2014_JSRT_70.8.773

[6] Sayed, I.S. and Mohamed Nasrudin, N.S. (2016) Effect of Cut-Off Frequency of Butterworth Filter on Detectability and Contrast of Hot and Cold Regions in Tc-99m SPECT. International Journal of Medical Physics, Clinical Engineering and Radiation Oncology, 5, 100-109. https://doi.org/10.4236/ijmpcero.2016.51011

[7] Tomasi, C. and Manduchi, R. (1998) Bilateral Filtering for Gray and Color Images. IEEE International Conference on Computer Vision, Bombay, 7 January 1998, 839-846. https://doi.org/10.1109/ICCV.1998.710815

[8] Zhou, J., Zhu, H., Shu, H. and Luo, L. (2007) A Generalized Diffusion Based Inter-Iteration Nonlinear Bilateral Filtering Scheme for PET Image Reconstruction. Computerized Medical Imaging and Graphics, 31, 447-457.

https://doi.org/10.1016/j.compmedimag.2007.04.003

[9] Hofheinz, F., Langner, J., Beuthien-Baumann, B., Oehme, L., Steinbach, J., Kotzerke, J., et al. (2011) Suitability of Bilateral Filtering for Edge-Preserving Noise Reduction in PET. EJNMMI Research, 1, 1-9. https://doi.org/10.1186/2191-219X-1-23

[10] Katayama, Y., Ueda, K., Hiura, S., Yamanaga, T., Miyoshi, H., Ohmura, M., et al. (2013) Bilateral Filter Applied to Bone Scintigraphy. Nihon Hoshasen Gijutsu Gakkai Zasshi, 69, 1363-1371. https://doi.org/10.6009/jjrt.2013_JSRT_69.12.1363

[11] Buades, A., Coll, B. and Morel, J.-M. (2005) A Non-Local Algorithm for Image Denoising. IEEE International Conference on Computer Vision and Pattern Recognition, 2, 60-65.

[12] Paris, S., Kornprobst, P., Tumblin, J. and Durand, F. (2008) Bilateral Filtering: Theory and Applications. Foundations and Trends in Computer Graphics and Vision, 4, 1-73. https://doi.org/10.1561/0600000020

[13] Maeda, H., Yamaki, N. and Azuma, M. (2012) Development of the Software Package of the Nuclear Medicine Data Processor for Education and Research. Nihon Hoshasen Gijutsu Gakkai Zasshi, 68, 299-306. https://doi.org/10.6009/jjrt.2012_JSRT_68.3.299

[14] Nagasawa, S. (2002) Improvement of the Scheffe's Method for Paired Comparisons. Kansei Engineering International, 3, 47-56. https://doi.org/10.5057/kei.3.3_47

[15] Hideyuki, T. (2014) Practical Statistical Tests Machine Learning (3) Significance Tests for Human Subjective Tests. Systems, Control and Information, 58, 514-520. 